\title{
Bit Error Rate (BER) Performance of MIMO Systems in M-QAM with Nonlinear Effect
}

\author{
Ahmed E. Zakzouk, Ragab M. Elbakar, and Mohamed I. Yousef
}

\begin{abstract}
Out object in this paper it to study, the effect of nonlinearity on the bit error rate (BER) of MIMO systems in $M-Q A M$ modulation techniques. We consider Saleh's model (power amplifier model) for the nonlinearity, and apply the nonlinear model on MIMO system with receiver diversity and transmitter diversity. For transmitter diversity, the Space-Time Block Coding (STBC) based on Alamouti scheme is used to provide transmits diversity for two transmitting antennas. The results show that, if there is a high variation in the amplitude of the M- QAM symbols, there will behigh effect of nonlinearity that causes high BER especially for high amplitude symbols at high SNR.
\end{abstract}

Keywords: Bit Error Rate, MIMO systems, Nonlinear Effect, Space-Time Block Coding.

\section{INTRODUCTION}

High quality (i.e. small BER and high bit rate data services) is predicted as the behavior of the next generation of wireless communications. Also, the units of remote are of low lightweight communicators that are to operate well in different circumstances.The time-varying multipath presents major phenomenon that causes difficulties in flexible cordless communication [1]. This phenomenon requires about $10 \mathrm{~dB}$ increasing in SNR to reduce the BER from 10-2 to $10-3$, which can be achieved by only $2 \mathrm{~dB}$ increasing in additive white Gaussian noise (AWGN). This SNR amelioration cannot be achieved either by higher transmit power or large bandwidth, as it is opposite to the requirements of next generation of wireless communication systems. Therefore, it is of interesting to effectively decrease the fading effect the base stations and at the remote units by using MIMO system, without additional power or any sacrifice in bandwidth [2]. Fortunately, for the purpose of the economy, more complex base stations that contain multiple antennas are allowed to be used. This multiple antenna system may be the only plausible trade space for achieving the requirements of next generation wireless systems.

Revised Manuscript Received on December 30, 2019.

* Correspondence Author

*Ahmed E. Zakzouk, Electrical Engineering Department, Collage of Engineering, Al-Azhar University, Cairo, Egypt.

Ragab M. Elbakar, Electrical Engineering Department, Collage of Engineering, Al-Azhar University, Cairo, Egypt.

MohamedI. Yousef, dElectrical Engineering Department, Collage of Engineering, Al-Azhar University, Cairo, Egypt.

(C) The Authors. Published by Blue Eyes Intelligence Engineering and Sciences Publication (BEIESP). This is an open access article under the CC BY-NC-ND license (http://creativecommons.org/licenses/by-nc-nd/4.0/)
In wireless communication systems, variable devices such as high power amplifiers, low-noise amplifiers, mixers, etc. can cause nonlinearity. The nonlinearity affects channel capacity and BER. To study the effect of the nonlinearity on the required MIMO system, we need to model its effect. One of the most important models of nonlinearity is Saleh's model that describes the dependence between the input and output powers by the (AM-AM) conversion characteristics. In addition, it describes the dependence between the input power and the output phase shift by the (AM-PM) conversion characteristics. Typical Saleh model characteristics are shown in Figure (1).

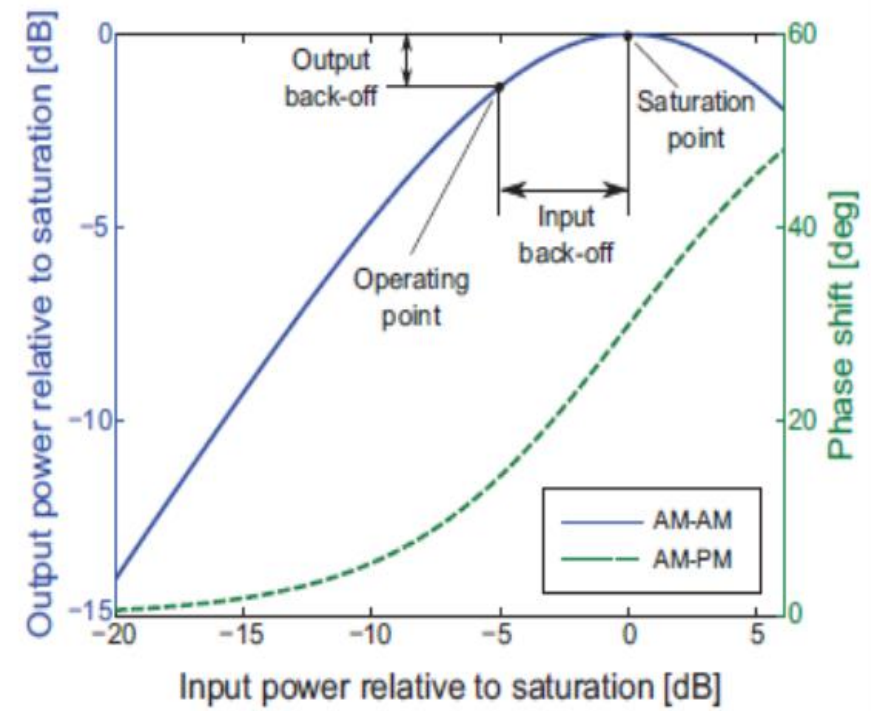

Fig. 1: Saleh's model for AM-AM and AM-PM
characteristic of Power Amplifier

\section{MIMO SYSTEM}

MIMO communication systems were studied for more than one decade and proved theoretically that they improve capacity, reliability, coverage, or combinations in comparison with different systems that have a mono antenna at the transmitter, the receiver or both of them [6] [7]. MIMO also has different advantages, such as beam forming gain, spatial diversity, and multiplexing. By using beam forming, transmit and receive antenna, patterns can be focused into a specific angular direction by choosing a complicated system of baseband antenna. In line-of-sight (LOS) conditions of operations and gains add up, causing an upper limit of $\mathrm{m} \cdot \mathrm{n}$ for the beam forming gain of a MIMO system ( $\mathrm{n}$ and $\mathrm{m}$ refer to the number of antennas of the receiver $\mathrm{Rx}$ and for the transmitter $\mathrm{T}_{\mathrm{x}}$ respectively). 


\section{Bit Error Rate (BER) Performance of MIMO Systems in M-QAM with Nonlinear Effect}

For a certain bandwidth, the goal is to increase the capacity in wireless systems. This motivated researchers to achieve a higher throughput on. One of the most important aspects of this research is that for a medium rich in multipath components, the wireless channel capacity can be augmented using multi antenna on both sides of the link. Comprehensive as a measurement of performance of STBC techniques in vital channels depends on precise information of the characteristics of spatiality of the cordless channel. To improve the possible gain attained by these systems, it is of detailed knowledge to know the matrix of MIMO channel. Scenarios that have this high capacity actually use the multipath structure by clever coding of the data in both space and time [8]. We consider BER as one of the measurements for MIMO; we simulate the effect of nonlinearity in receiver diversity model $1 \times 2$, Alamouti $2 \times 1$ space time coding model and No diversity model.

\section{SPACE TIME BLOCK CODING?}

Alamouti presents the basic part of STBC [2] [9]. This technique gives a diversity of transmit to MIMO system (Fig.2). In this figure, a maximum Ratio Receive Combining (MRRC) scheme is shown. The outline scheme uses a pair of transmit antennas and a received one and has the following characteristics:

- A transmission sequence information Symbols are encoded at the transmitter

- Signals and noise at the receiver are Combined

- A detection is achieved by Maximum likelihood During a specified symbol period, two different symbols are transmitted at the same time from the two antennas by Alamouti STBC. In this situation, the first symbol in the sequence, $S_{1}$, is transmitted from the one antenna 1 while the second one, $S_{2}$, is simultaneously transmitted from the other antenna on the other hand, in the subsequent symbol time, the signal $-\mathrm{S}_{2} *$ is transmitted from the one antenna and the signal $S_{1} *$ is transmitted from other antenna.

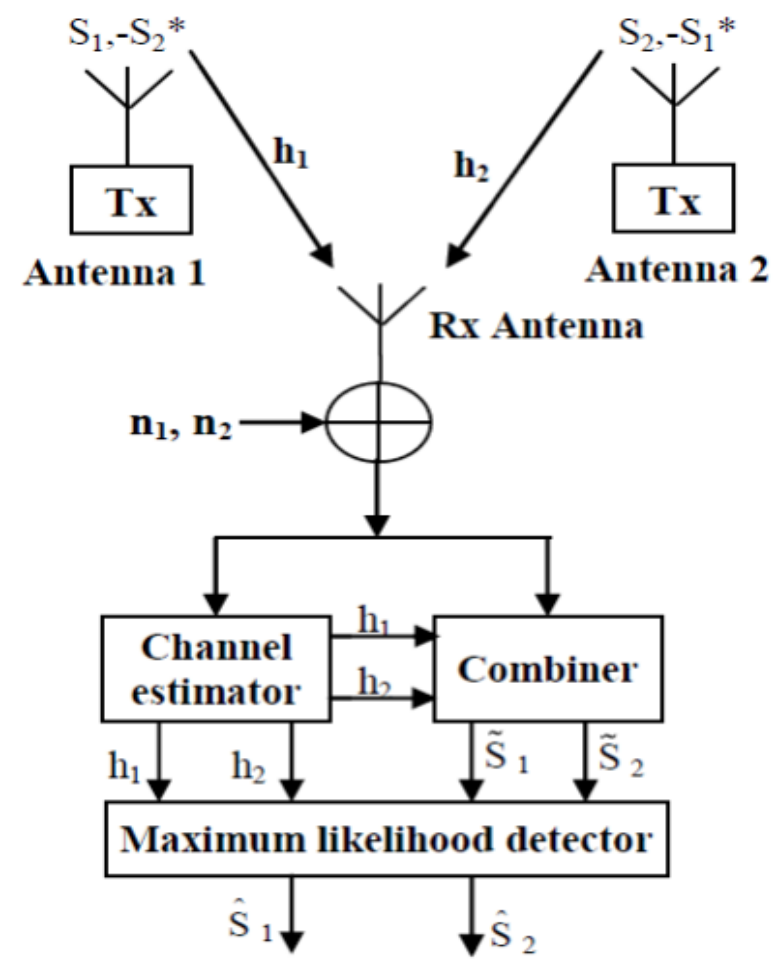

Fig.2: Alamouti's Model

Mathematically, the obtained signals can then be formulated as:

$$
\begin{array}{r}
\mathrm{r}_{0}=\mathrm{r}(\mathrm{t})=\mathrm{h}_{1} \mathrm{~s}_{1}+\mathrm{h}_{2} \mathrm{~S}_{2}+\mathrm{n}_{1} \\
\mathrm{r}_{1}=\mathrm{r}(\mathrm{t}+\mathrm{T})=-\mathrm{h}_{1} \mathrm{~S}_{2} *+\mathrm{h}_{2} \mathrm{~S}_{1} *+\mathrm{n}_{2}
\end{array}
$$

Where:

$\mathrm{r}_{0}$ and $\mathrm{r}_{1}$ denote the received signals at time $\mathrm{t}$ and $\mathrm{t}+\mathrm{T}, \mathrm{n}_{1} \& \mathrm{n}_{2}$ represent receiver noise and interference complex random variables. The pair of signals $S_{1}$ and $S_{2}$ is then transmitted through the maximum likelihood detector.

$\mathrm{S}_{1}=\mathrm{h}_{1} * \mathrm{r}_{0}+\mathrm{h}_{2} \mathrm{r}_{1}$

$\mathrm{S}_{2}=\mathrm{h}_{2} * \mathrm{r}_{0}-\mathrm{h}_{1} \mathrm{r}_{1} *$

At the receiver, the actual transmitted symbols, $S_{1}$ and $S_{2}$ are detected. A good knowledge of the channel coefficients, $\mathrm{h}_{1} \& \mathrm{~h}_{2}$ is required; these coefficients are estimated at the receiver

\section{SYSTEM MODEL}

In figure.1, the model of MIMO is shown. By using M-QAM digital modulation techniques, the incoming data stream is modulated. This data is transmitted viaSTBC. These modulated data stream are passed through the channel which contains nonlinear amplifier and then first decoded by STBC decoder and after that detected by digital demodulator of M-QAM to obtain the received signal.

A random source is used to generate a series of ones and zeros as data stream. After generation of data, this data is moved to the next stage of symbol mapping [10]. Three modulation techniques are used for digital modulation which includes4- QAM, 16-QAM, and 256-QAM.

The received signal "y", that passing through the nonlinear channel, is obtained by the next equation:

$y=H f(s)+n$

Where: 
$H$ is the channel matrix of MIMO, $\mathrm{n}$ is the noise array, and $f(s)$ is a function that describes the nonlinearity of the modulated symbol $s$ that affected by the HPA.

In figure 1, a HPA is plotted regarding the AM/AM (amplitude-to-amplitude distortions) and AM/PM (amplitude-to-phasedistortions) characteristics.

The output of the HPA, using Saleh model, is given by [4]:

$f(s)=s \frac{A(r) e^{j \phi(r)}}{r}$

Where:

Sis the input symbol vector that is modulated to the HPA, $r=$ $|s|$ and $|$.$| is the magnitude of the s$. The A(r) (AM/AM) and $\varphi(\mathrm{r})(\mathrm{AM} / \mathrm{PM})$ characteristics given in (5) are defined as:

$A(r)=\frac{\alpha_{A M} r}{1+\beta_{A M} r^{2}} \quad \emptyset(r)=\frac{\alpha_{P M} r}{1+\beta_{P M} r^{2}}$

Where:

$\alpha_{A M}, \beta_{A M}, \alpha_{P M}$, and $\beta_{P M}$ are the power amplifier parameters

\section{RESULT AND DISCUSSION}

In this part, we show the system simulation results to study the effect of nonlinearity due to HPA (Saleh's model) on the BER of MIMO systems. We simulate the M-QAM modulation over receiver diversity $1 \times 2$ MIMO with maximum ratio receiver combining (MRRC),

over $2 \times 1$ transmitter diversity MIMO using Alamouti system, and over no diversity (single input single output) system.

In figure 3, the BER of 4- QAM modulation is shown for the different systems (assuming linear and nonlinear). The results show that the nonlinearity has minor effect on the BER performance of the 4-QAM modulation. The 4-QAM symbols are not affected by the nonlinearity distortion, because the variation in its amplitude does not exist.

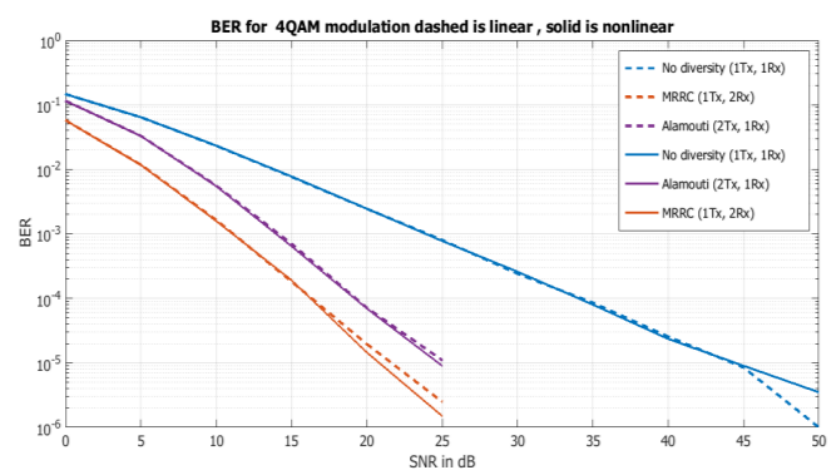

Fig. 3: BER against SNR for 4-QAM

In figure 4, BER of the system shows clear difference between linear and nonlinear for 16-QAM modulation in high SNR, i.e. BER for linear is smaller than nonlinear, due to the power amplifier is in saturation region, where the nonlinearity is increased. In low SNR the nonlinear is closed to linear, because in low SNR the power amplifier is in linear region and nonlinearity effect is not exist.

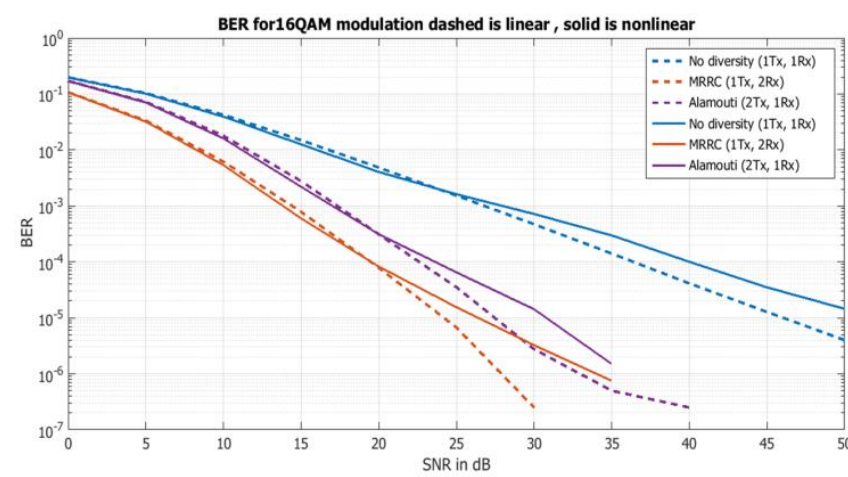

Fig. 4: BER against SNR for 16-QAM

The large difference in BER between linear and nonlinear systems in 256-QAM is shown in figure 5. For 256 QAM,we have high variations in signal amplitude (not equal) that have a high effect of nonlinearity, that caused high BER specially for high amplitude symbols.

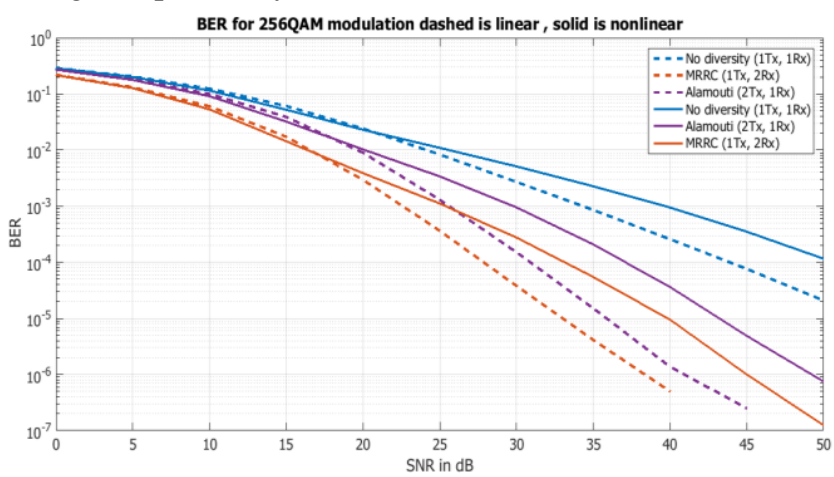

Fig. 5 BER against SNR for 256-QAM

\section{CONCLUSION}

In this research work, we have studied the nonlinearity effect caused by HPA on BER of MIMO systems. We found that that the nonlinearity has a minor effect on the BER performance of the 4-QAM modulation but a considerable effect on the BER performance of the other two modulations more prominent in 256-QAM modulation.

\section{REFERENCES}

1. W.C. Jakes, "Microwave Mobil Communications". New York: Wiley. 1974

2. S.M. Alamouti" A Simple transmits diversity Techniques for Wireless Communications" IEEE Journal on Select Areas in communications, Vol. 16 No. 8, Oct. 1998.

3. Khaled M. Gharaibeh, "Nonlinear Distortion in wireless Systems" John Wiley. 2012

4. A. A. M. Saleh, "Frequency-Independent and Frequency- Dependent Nonlinear Models of TWT Amplifiers," IEEE Trans. Commun., vol. COM-29, pp. 1715-1720, Nov. 1981.Trans. Comm. 29, 1715-1720.

5. Dantona, V.; Delamotte, T.; Bauch, G.; Lankl, B.: Impact of nonlinear power amplifiers on the performance of precoded MIMO satellite systems. International IEEE-AESS Conference in Europe about Space and Satellite Telecommunications (ESTEL Conference), Rome, Italy, October 2-5, 2012.

6. Steven Howard, HakanInanogle, and John Ketchi" Result from MIMO channel Measurements" IEEE international Conference, 2002.

7. Jerry R. Hampton, Manuel A, Cruz, "MIMO Channel Measurements for Urban Military Applications" IEEE international Conference 2008.

8. KhatendraYadav "CHANNEL ESTIMATION IN MIMO WIRELESS ENVIRONMENT" 2014. 
Bit Error Rate (BER) Performance of MIMO Systems in M-QAM with Nonlinear Effect

9. Shubhangi Chaudhary, and A.J. Patil "PERFORMANCE ANALYSIS OF MIMO-SPACE TIME BLOCK CODING WITH DIFFERENT MODULATION TECHNIQUES" ICTACT Journal

Communication Technology, March, 2012, Vol.03, Issue 01.

10. J.G. Andews, A. G. (2007). Fundamentals of WiMAX Understanding Broadband Wireless Networks. Prentice Hall.

\section{AUTHORS PROFILE}
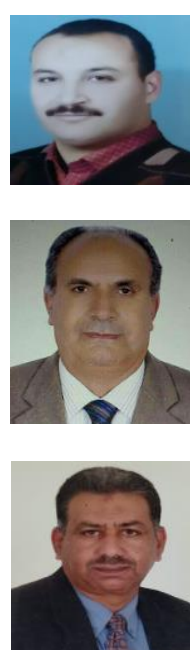

Ahmed E. Zakzoukwas born in Al-Sharkia, Egypt in 1972. He received the B.S. and M.S. degrees in electrical engineering from the Faculty of Engineering, AlazharUniversity, Cairo, Egypt, in 1996, and 2007 respectively. His research activities include analog and digital communication systems.

Ragab M. Elbakarwas born in Cairo at 1961. He received the B.S.M.S. and $\mathrm{PhD}$. degrees in electrical engineering from the Faculty of Engineering, AlazharUniversity, Cairo, Egypt, in 1986,19942003and respectively. His research activities include analog and digital communication systems.

Mohamed I. Youssef received the Dr.Ing. degree from Ruhr University Bochum, Germany in 1988. He is a professor of telecommunications at $\mathrm{Al}$-azhar University in Cairo, Egypt. His research interests include the digital signal processing, wireless communications, nonlinear systems and the algorithms of digital signal processing. 